relationship, (30.7\%) reported they would still have sex with their most recent partner yet $95 \%$ stated they currently do not have a monogamous relationship with this partner.

Conclusions Female adolescents with abuse histories who describe relationship status as not involved or involved with more than one partner engage in more risk behaviour than those who report exclusive relationships with one partner. Explicating behaviour that occurs by relationship status has implications for interpreting and addressing risk for intervention and practice guidelines.

\section{P2-S9.13 AFRICAN- AND MEXICAN-AMERICAN ADOLESCENT WOMEN WITH STI AND A HISTORY OF ABUSE: BIOLOGICAL OUTCOME OF A RANDOMISED TRIAL OF BEHAVIOURAL INTERVENTION}

doi:10.1136/sextrans-2011-050108.399

J D Champion, J Collins. Texas Tech University, Health Science Center, Lubbock, USA

Background To implement a randomised trial of a risk-reduction intervention consisting of small group sessions, individual counselling and support groups for African- and Mexican-American adolescent women with STI and abuse history and evaluate the effects of the intervention model vs enhanced counselling for this group on STI at 6 and 12 month follow-up.

Methods Mexican- and African-American adolescent women ( $n=409,13-18$ years) with STI and a history of abuse (physical, psychological, sexual) were recruited from public-health clinics in a metropolitan area of the USA. Following enrolment participants received targeted physical exams including contraception counselling and interviews including assessments for abuse, sexual risk behaviour, substance use, contraceptive use and STI. Participants were encouraged to return for unscheduled visits as needed for counselling or suspected STI or pregnancy. Scheduled follow-up rates at $6(93 \%)$ and $12(93 \%)$ months were high Participation in risk-reduction interventions was also high (92\%). Surveillance for STI and pregnancy at off-site clinic visits was conducted and documented throughout the study.

Results At study entry, participants reported sexual (58.9\%), physical (76.8\%) and psychological (82.4\%) abuse histories, ever pregnant (45\%), currently pregnant $(18.7 \%)$, ever used birth control $(29.9 \%)$, currently in school (63.2\%), previous arrest $(56.2 \%)$, home runaway $(54.5 \%)$, any substance use $(94.1 \%)$ including marijuana $(82.6 \%)$, heroin $(13.4 \%)$, cigarettes $(74.6 \%)$, alcohol $(77.5 \%)$, cocaine $(41.1 \%)$ and benzodiazepine (45\%); forced sex $(29.1 \%)$, sex when high/out of control $(13.9 \%)$, sex with friends for benefits $(36.2 \%)$, steady partner in last 3 months (77.4\%) however off and on (31.4\%). More workshop vs control group participants reported physical abuse $(82.9 \%$ vs $71.0 \%)$, home runaway $(63.1 \%$ vs $46.4 \%)$, cigarettes $(78.9 \%$ vs $70.5 \%)$ and alcohol use ( $81.4 \%$ vs $73.8 \%)$. More control group participants were currently in school ( $68.1 \%$ vs $58.1 \%)$ and had used condoms (95.3\% vs $86.4 \%)$. These variables plus age/ethnicity were initially included in the regression model for analysis of data from 0 to 6 and $0-12$ month follow-ups. Fewer STI re-infections for intervention vs control group participants at each study interval were identified; physical abuse and substance use remained in the model.
Conclusions Behavioral interventions may reduce of STI among African-and Mexican-American adolescent women with STI and a history of abuse.

\section{P2-S9.14 A QUALITATIVE, LONGITUDINAL STUDY OF POST- DIAGNOSIS REACTIONS AMONG HSV-2 SEROLOGIC POSITIVE WOMEN}

doi:10.1136/sextrans-2011-050108.400

${ }^{1} \mathrm{~J}$ E Brand, ${ }^{2}$ B Van Der Pol. 'Marion County Health Department, Ball State University, New Palestine, USA; ${ }^{2}$ Indiana University Medical Center \& IU-Bloomington, Bloomington, USA

Background Herpes simplex virus type 2, the main cause of genital herpes, is found worldwide among populations. US National seroprevalence is estimated at $16.2 \%$, with highest rates among women (20.9\%) \& non-Hispanic blacks (39.2\%). Medical consequences of HSV-2 infection includes a two- to five-fold increased risk for HIV 1 transmission \& neonatal herpes. The advent of type-specific HSV serologic tests offers accurate methods of diagnosis for those who are asymptomatic. However, diagnosis of HSV-2 has been noted to be distressing for those who are asymptomatic \& unaware of infection. The purpose of this study is to understand social \& emotional impact of HSV-2 serodiagnosis on asymptomatic women over time.

Methods Purposeful sampling was conducted \& 28 women, newly diagnosed as HSV-2 serologic positive \& asymptomatic, were recruited from a Midwestern STD clinic \& urban community court. A series of three open-ended interviews were conducted over 6-month period. Interviews were audio recorded \& transcribed. Important areas explored: emotional \& social responses to diagnosis; motivations for (non) disclosure of HSV-2 status; exploration of sexual behaviour post-diagnosis; use of condoms \& suppressive therapy. Qualitative analysis was done using manual coding.

Results Age of participants ranged from 19 to 61 yrs. Majority were African-American (71\%) with 21\% white. No participants reported knowledge of HSV-2 status at diagnosis. Five themes emerged during analysis of first interviews: rumination \& disclosure anxiety; knowledge deficit anxiety; stigmatisation \& alteration in selfconcept; fear/apprehension regarding future; impact on sexuality \& partnering. With analysis of 6-month interviews there was an iteration of two themes-alteration in self-concept \& impact on sexuality \& partnering. Of 23 participants who completed three interviews-22\% had no plans for sex after diagnosis, 39\% never disclosed HSV-2 status to partners \& $56 \%$ never used suppressive therapy.

Conclusions Findings suggest that despite increased public information related to HSV-2, initial diagnosis remains traumatic, \& for a small percentage anxiety lingers for at least 6 months. Providers should be aware of need for written information targeted to nonclinicians \& that further follow-up should be initiated after diagnosis to reinforce learning, clarify concerns, counsel \& support. Providers should plan additional time for client integration of diagnosis, implications \& questions. 